\title{
The role of absorption and reflection in the soft X-ray excess of Active Galactic Nuclei
}

\section{Preliminary results}

\author{
L. Chevallier ${ }^{1}$, S. Collin ${ }^{1}$, A.-M. Dumont ${ }^{1}$, B. Czerny ${ }^{2}$, M. Mouchet ${ }^{1,3}$, \\ A. C. Gonçalves ${ }^{1,4}$, and R. Goosmann ${ }^{1}$ \\ ${ }^{4}$ Centro de Astronomia e Astrofísica da Universidade de Lisboa, Observatório Astronómico de Lisboa, Tapada da Ajuda, 1349-018 Lisboa,
}

1 LUTH, Observatoire de Paris, Section de Meudon, 92195 Meudon Cedex, France e-mail: loic.chevallier@obspm. fr

2 Copernicus Astronomical Center, Bartycka 18, 00-716 Warsaw, Poland

3 APC, Université Denis Diderot, 75005 Paris, France Portugal

Received 30 June 2005 / Accepted 21 October 2005

\section{ABSTRACT}

The $2-10 \mathrm{keV}$ continuum of AGN is well represented by a single power law, generally attributed to a hot Comptonizing medium, such as a corona above the accretion disk. At lower energies the continuum displays an excess with respect to the extrapolation of this power law, called the "soft X-ray excess". Until now it was attributed either to reflection of the hard X-ray source by the accretion disk or to the presence of an additional Comptonizing medium. An alternative solution is that a single power law correctly represents both the soft and the hard X-ray emission, and the soft X-ray excess is an artefact due to the absorption of the primary power law by a relativistic wind. We examine the advantages and drawbacks of the reflection versus absorption models. We argue that in the absorption hypothesis, the absorbing medium should be in total pressure equilibrium to constrain the spectral distribution which otherwise would be too strongly variable in time and from one object to the other, as compared to observations. We conclude that some X-ray spectra, in particular those with strong soft X-ray excesses, can be modelled by absorption in the $0.3-10 \mathrm{keV}$ range. However, due to the lack of a complete grid of models and good data extending above $10 \mathrm{keV}$, we are not able to conclude that all objects can be accommodated by such models. These absorption models imply either strong relativistic outflowing winds with mass rates of the order of the Eddington value (or even larger), or quasi-spherical inhomogeneous accretion flows. Only weak excesses can be modelled by reflection, unless the primary continuum is not directly seen. A reflection model absorbed by a modest relativistic wind could be the best solution to the problem.

Key words. quasars: general - galaxies: Seyfert - X-rays: general

\section{Introduction and rationale}

The X-ray spectra of radio quiet Active Galactic Nuclei (AGN) are well represented by one or more broad continuum components with superimposed spectral features due to absorption and emission by the circumnuclear material. The key problem is that the decomposition of the observed spectrum is not unique.

The important role of both the emission and the absorption features in shaping the observed spectra is clear. Significant intrinsic absorption was measured in Ariel 5 observation of NGC 4151 (Ives et al. 1976). The first X-ray narrow absorption feature was detected by Halpern (1984) in EINSTEIN data. Now more than $50 \%$ of well studied Seyfert galaxies and many quasars are known to possess absorbers at various ionization degrees (e.g. Seyfert 1: Nicastro et al. 2000;
Behar et al. 2003; Steenbrugge et al. 2005; Young et al. 2005; Narrow Line Seyfert 1: Leighly et al. 1996; Gallo et al. 2004; PG QSO: Pounds et al. 2003a,b; Porquet et al. 2004; Piconcelli et al. 2004, 2005; BAL QSO: Gallagher et al. 2002; Grupe et al. 2003). The first X-ray emission feature - the Fe fluorescent line - was measured in 1978 (Mushotzky et al. 1978, Cen A). Later, the Compton reflection component was identified in a composite Ginga 12 spectrum (Pounds et al. 1990), and a broad Fe line was found (Tanaka et al. 1995) consistent with the expectations of a relativistically broadened feature due to X-ray reprocessing by an accretion disk. Nowadays many emission lines are identified in Seyfert 2 spectra (e.g. Sambruna et al. 2001; Kinkhabwala et al. 2002), iron lines are seen in most Seyfert 1 and 2 galaxies as well as in many quasars, and a few broad soft X-ray lines were possibly identified (Kaastra et al. 2002; Różańska et al. 2004). Relatively narrow features come from a 
significantly ionized medium, called the warm absorber (hereafter WA), which is outflowing with velocities from hundreds to thousands $\mathrm{km} \mathrm{s}^{-1}$ from the nuclear region (cf. for instance Blustin et al. 2005 for a collation and an analysis of the results concerning 23 objects). This WA is located most probably somewhere between the Broad and the Narrow Line Region.

However, determining whether a given feature is due to absorption or emission is not always simple. There are two main issues which are still under discussion:

- the question of whether objects like Mkn 766 possess strong and relativistically smeared soft X-ray emission lines or the observed feature is actually due to the (dusty?) warm absorber (Branduardi-Raymont et al. 2001; Turner et al. 2003; Sako et al. 2003);

- the nature of the apparent slope change in the overall X-ray spectrum at $\sim 1 \mathrm{keV}$ in Seyfert 1 galaxies and radio quiet quasars. If the X-ray spectrum of an object is fitted with a power law plus absorption and eventually some components as the Compton reflection, the iron line or narrow spectral features, the model usually underpredicts the observed spectrum in the soft X-ray range. An additional component - a soft X-ray excess - is needed (Wilkes \& Elvis 1987). The spectral shape and the nature of this component has been under discussion for many years (e.g. Czerny \& Zycki 1994). This component is usually modelled either as an additional continuum component (black body or multicolor blackbody; bremsstralhlung: Barvainis 1993; thermal Comptonization: Magdziarz et al. 1998), or as a strongly ionized reflection (i.e. scattering radiation with many atomic features like emission lines and recombination continua: Ross \& Fabian 1993, and subsequent works). However, as shown by Gierliński \& Done (2004), this apparent change of slope is equally well modelled as being due to absorption of an originally rather soft power law intrinsic spectrum due to the warm absorber.

Resolving these issues is essential both to understand the behaviour of the absorbing nuclear material and for the determination of the true intrinsic spectrum and, subsequently, to understand the process of accretion flow onto the central black hole.

The presence of material surrounding the central source affects the observed spectrum, and in particular leads to both emission and absorption features. The effect depends on the location of the medium, its ionization, clumpiness, as well as the specific line of sight to the source. Some of the plasma parameters cannot be taken as arbitrary since the thermal state of the material is determined by the physical conditions.

The aim of this paper is to consider the advantages and drawbacks of the reflection versus absorption models, by modeling the transfer of radiation in the plasma surrounding the central black hole, taking into account its natural physical limitations.

In the next section, we recall some generalities about the models and our photoionization code. In Sect. 3 we consider pure absorption models, and we show that, unless a constraint of total pressure equilibrium is imposed, they should lead to

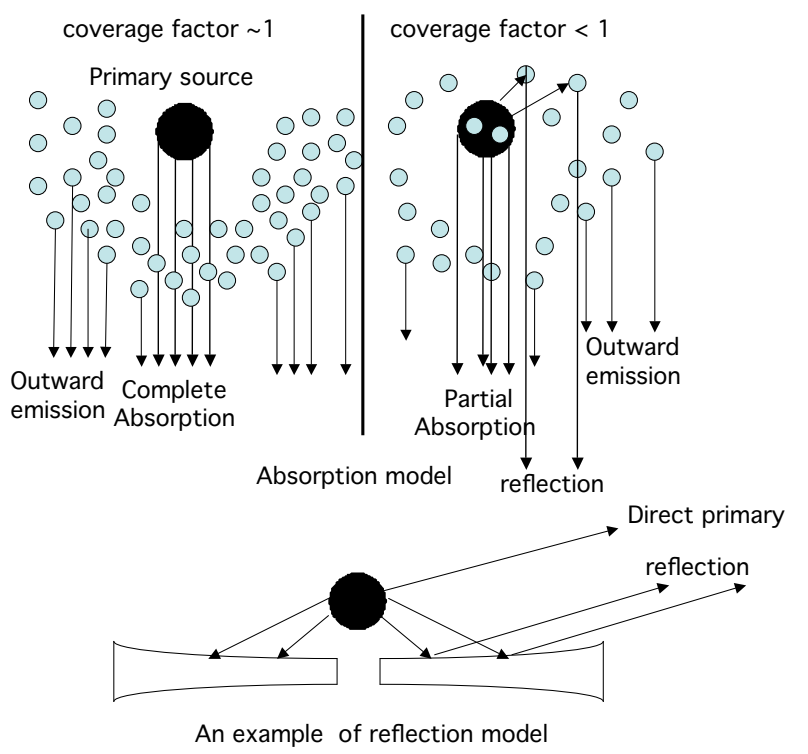

Fig. 1. Scheme of the absorption and reflection models showing the combination of spectra that constitutes the observed spectrum.

a very large variation of the absorption spectrum. In Sect. 4 we consider intermediate models (partial coverage and absorption/emission), then reflection models, and we show that the observed spectrum is mainly a function of the coverage factor and of the column density. Finally, in the last section, we discuss some physical implications of these models.

\section{Some generalities}

AGN are characterized by an X-ray spectrum extending into the gamma ray range up to hundred $\mathrm{keV}$, or several hundred $\mathrm{keV}$. This emission is assumed to be provided by a region located close to the central black hole. Any medium surrounding this primary source is radiatively heated and photoionized and will reprocess the primary photons as soft X-rays, UV, and possibly optical and IR photons. Whatever the structure of this medium - clumpy or continuous - the observed spectrum will thus be a combination of "reflection" by the illuminated side (which is actually not a real reflection, as it includes atomic and Compton reprocessing), "outward emission" (emission by the back side), transmission of the incident primary spectrum, and possibly the primary spectrum itself. The amount of each component depends on the coverage factor of the source by the medium and on its geometry (see Fig. 1).

If the irradiated medium has a flat and continuous structure, like an accretion disk, one will see a combination of about equal proportions of primary and reflected radiation. This is the most simple case, because if the medium is distributed quasispherically, all components should be observed, although one or two of them can be negligible. If its coverage factor is very close to unity, only the outward emission plus transmission will be observed. If the coverage factor is small, the primary source plus the reflection and the outward emission will be observed.

Thus only in some cases will a single component be observed: a pure absorption spectrum requires the source to be completely covered and a small column density, in order for 
the outward emission to be negligible. It is indeed difficult to imagine that the line of sight is completely covered, and that the other directions are not covered; it would mean that the absorbing medium is confined in a very small cone or filament along the line of sight. This is certainly neither the case of the WA which is observed in a large fraction of Seyfert 1s, nor the Gierliński \& Done (2004) model, which is assumed to account for the soft X-ray excess in all bright quasars. A pure reflection spectrum requires a peculiar disposition of the medium, as in the inhomogeneous accretion disk model proposed by Fabian et al. (2002, see the discussion below).

All components are sensitive to several parameters. The thermal and ionization structure is determined mainly by the shape of the continuum in the soft X-ray range and by the ionization parameter. It is also not often realized that the upper and lower energies of the incident continuum play an important role in determining the free-free (at high density) and the Compton heating and cooling, as well as the ionization states of the elements. The absorption spectrum depends strongly on the column density, since the cool absorbing layers are located at the back side of our medium. The metal abundances influence not only directly the line spectrum but determine also the structure of the medium. On the contrary, micro-turbulence has a strong impact only on the line intensities in emission or in absorption, but a small influence on the thermal and ionization structure, at least for high column densities where the cooling is dominated by bound-free transitions. Also the density is not important, except for the relative intensities of the forbidden and permitted emission lines (for instance those of the helium-like ions like O VII), and the overall spectrum is almost the same for a gas density varying from $10^{7}$ to $10^{12} \mathrm{~cm}^{-3}$ (Różańska et al. 2006). This is why we do not specify the density used in the models, and why the computed fluxes in the figures are always given in arbitrary units (they are proportional to the density).

In this study, we used our photoionization code TITAN initially designed for ionized thick media (Thomson thickness up to several tens). The code is equally suited to model thinner media like the WAs. Its advantage over the other photoionization codes like Cloudy (Ferland et al. 1998), XSTAR (Kallman \& Bautista 2001), or ION (Netzer 1993, 1996) is that it treats the transfer of both the lines and the continuum using the powerful ALI method (Accelerated Lambda Iteration), which allows us to compute very precisely line and continuum fluxes. The other photoionization codes use, at least for the lines, an integral formalism called the "escape probability approximation". In particular the computation of the absorption and emission lines is uncoupled when using this approximation, while with ALI both the lines and the continuum are treated in a consistent way. In the context of X-ray spectra of AGN, the escape probability approximation can lead to errors by a factor of several units on the line intensities. More important for the present study, in the case of thick media, it leads to large errors in the temperature and ionization structure near the back side of the medium which gives rise to the absorption features (Dumont et al. 2003, and Collin et al. 2004).

TITAN has been described in several papers (for instance Dumont et al. 2000, 2003). We recall only that it solves the transfer of about 1000 lines and of the continuum, and gives as output the ionization and temperature structures, and the reflected, emitted outward, and absorbed spectra. The sophisticated line transfer treatment leads to a longer computation time than with other approximate methods (e.g., escape probabibility). Thus, our choice was to take fewer lines into account than available in Cloudy, XSTAR, or ION codes, so in particular for the WA it misses some important features (like the Unresolved Transition Array - UTA - around $750 \mathrm{eV}$, and the inner shell transitions, except the iron $\mathrm{K}$ lines). Nevertheless it is possible to improve atomic data and it is planned for future developments of our code. We show below that this lack of lines is not critical for such a study.

The previous versions of our code used a 2-stream approximation for the transfer, which is equivalent to the ALI method with a 1-point angular quadrature $(\operatorname{cosine}=1 / \sqrt{3})$. This approximation is consistent with a semi-isotropic illumination. For the study of the pure absorption spectrum corresponding to a normal illumination, it was necessary to first perform a computation with the 2-stream code to obtain the opacities and optical thicknesses of the model. Hence normal absorption was computed by the extinction of a normal incident flux for the same ionization parameter using the optical thicknesses obtained earlier (Różańska et al. 2006). A recent improvement was added to TITAN: to avoid this complex operation, we now use ALI in a multidirection version allowing us to take into account a normal or inclined illumination. One can then determine the emitted or reflected intensity as a function of the direction. The advantage is to obtain the emission and absorption spectra in a completely consistent way for a given opening angle and coverage factor of the medium.

Our models consist of plane-parallel slabs illuminated on one side by an incident continuum. In the majority of the following models, the incident radiation field is concentrated in a small pencil normal to the slab. The value of the pencil opening angle is not important, provided it stays much smaller than a fraction of a radian. Otherwise the absorption spectrum is mixed with emission (cf. Sect. 4).

Finally, a basic ingredient of the models is the ionization parameter $\xi$. Its definition varies among authors. We adopt $\xi=L / n_{\mathrm{H}} R^{2}$ (in the following $\xi$ will be given always in erg $\mathrm{cm} \mathrm{s}^{-1}$ ), where $n_{\mathrm{H}}$ is the hydrogen number density at the illuminated surface, $R$ the distance between the primary continuum source and the photoionized medium, and $L$ is the luminosity of the continuum. In the following we integrate $L$ over the whole primary continuum ( $10 \mathrm{eV}$ to $100 \mathrm{keV}$ in this study), but some authors prefer to integrate the luminosity only over the $0.1-10 \mathrm{keV}$ range where most of the X-ray absorption takes place, or from 0.54 to $10 \mathrm{keV}$, the energy range relevant for oxygen absorption, or from 1 to 1000 Rydberg as XSTAR does. The integration range should thus be taken into account when comparing the results of different authors.

\section{Pure absorption models}

\subsection{Constant density models}

In their paper, Gierliński \& Done (2004) modelled the X-ray spectrum of the highly accreting source PG 1211+143 (a 
Narrow Line Seyfert 1, or NLS1) by a pure absorption spectrum produced by an ionized slab of constant density located on the line of sight of a source with a steep power law continuum between 0.1 and $20 \mathrm{keV}$. The photon index $\Gamma$ is equal to 2.7 , quite a high value compared to typical observed indexes in the high energy range, but representative of radio quiet quasars and NLS1 in the soft X-ray band. The slab has a column density, $N$, equal to $3.3 \times 10^{23} \mathrm{~cm}^{-2}$, a turbulent velocity of $100 \mathrm{~km} \mathrm{~s}^{-1}$, cosmic abundances, and $\xi=460$ (these latter informations were kindly provided to us by C. Done). In order to get a "quasicontinuum" with no narrow features, Gierliński \& Done assumed that the lines and the photoelectric edges are smeared by a large velocity dispersion $v$, so they convolved the absorbed spectrum with a Gaussian velocity dispersion $v / c=0.2$ $(F W H M=2 \sqrt{2 \ln 2} v / c \sim 2.35 v / c)$ corresponding to a spectral resolution $R=1 /(2.35 v / c) \sim 2$ (definition of $R$ used throughout this study). This high velocity can be due to an accelerated outflow, or to a disk wind dominated by Keplerian motion; in the latter case it should be produced very close to the black hole, at a distance around $(c / v)^{2}=25$ gravitational radii $R_{\mathrm{G}}\left(R_{\mathrm{G}}=G M / c^{2}\right)$. Such a model fits the data without any separate soft excess. Also Sobolewska \& Done (2005) have fitted the spectrum of another Narrow Line Seyfert 1 (1H 0707-495) with an absorption model.

We have built a set of models made of slabs of constant density, illuminated by power law continua, and we have computed the pure absorption spectra, assuming that there is no corresponding emission and that the slabs completely cover the primary source of radiation $\left(f_{\mathrm{cov}} \sim 1\right)$. We illustrate the discussion first in Fig. 2 by a comparison between XSTAR and TITAN to get an idea of the influence of atomic data in our code, specially the missing UTAs. Using similar physical conditions as in Gierliński \& Done (2004), with $\xi=2350$ for the incident flux from $10 \mathrm{eV}$ to $100 \mathrm{keV}$, both codes provide similar results. The upper panel shows the incident and transmitted spectra for a high resolution ( 1000), and the lower panel corresponds to those spectra being convolved with a dispersion velocity $v / c=0.2$ (i.e, spectral resolution 2). Since the TITAN code takes into account the electron scattering while this effect is neglected in XSTAR, the TITAN spectrum for the same incident flux is systematically below the XSTAR spectrum by a factor given by the Thomson optical depth of the medium, and the incident and transmitted fluxes at $200 \mathrm{eV}$ are not equal in TITAN but equal in XSTAR. Therefore, for a better comparison of the effect of absorption on the spectra, we adjusted the XSTAR spectrum to the TITAN spectrum at $200 \mathrm{eV}$ for the continuum. For these convolved spectra, the differences are weak (less than 40\%), and could be explained notably by the different approaches in the treatment of radiative transfer and energy balance. Also the influence of the atomic data, clearly visible at spectral resolution 1000 specially for the UTAs region around $900 \mathrm{eV}$, is weak for such a smearing. This comparison illustrates how much these models are sensitive to the computational methods, independently of the parameters (see also the comparison between several codes in Pequignot et al. 2001). We note however that for both models we see only one trough, located at around $2 \mathrm{keV}$, the absorption below $400 \mathrm{eV}$ and above $8 \mathrm{keV}$ is the same, and the difference for the width
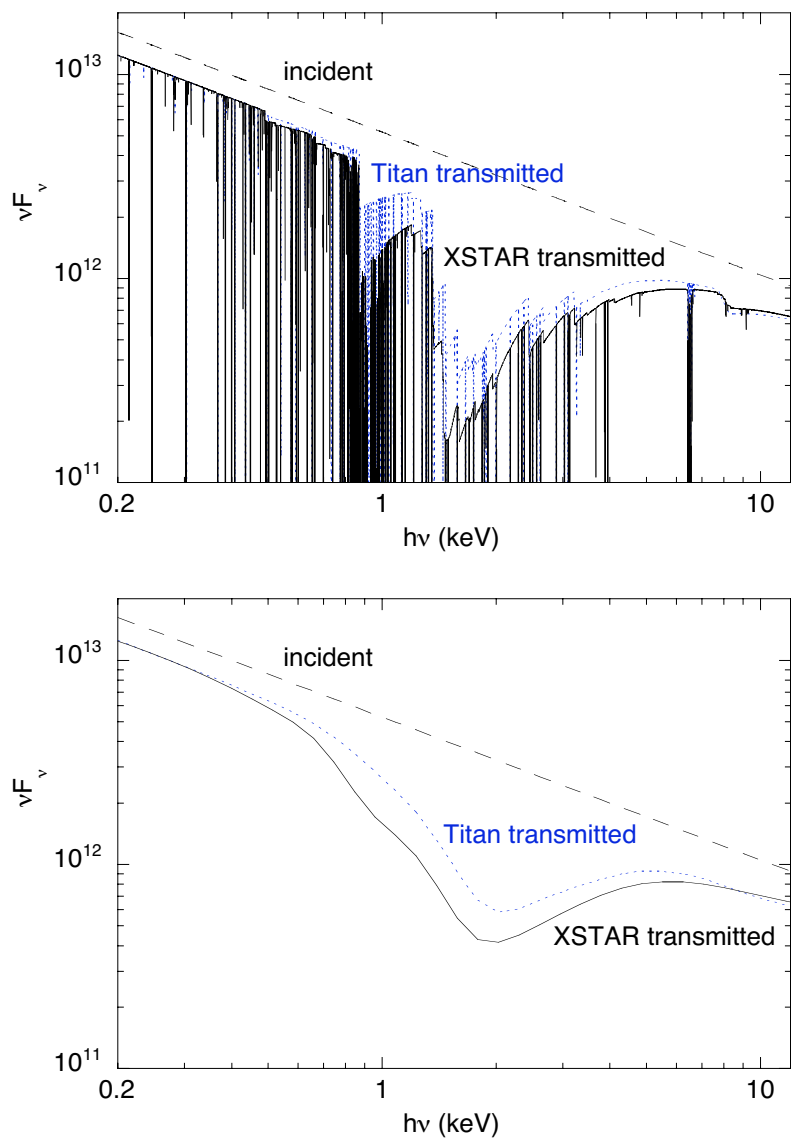

Fig. 2. Comparison of XSTAR and TITAN constant density calculations for physical conditions close to the Gierliński \& Done (2004) best fitting model to PG 1211+143. Both panels represent the primary continuum (black dashed lines) and transmitted fluxes (TITAN: blue dotted lines, XSTAR: black solid lines) $v F_{v}$ (arbitrary units) vs. energy (in eV). The spectra are given at a spectral resolution 1000 (upper panel) - differences for absorption lines, specially UTAs, are clearly visible - and convolved with a velocity dispersion $v / c=0.2$ corresponding to a spectral resolution 2 (lowel panel). Differences of fluxes are less than $40 \%$, and less than $10 \%$ for the width of the trough taken at half maximum.

of this trough (taken at half maximum) is less than $10 \%$. UTAs included in XSTAR are the reason for a second trough around $800 \mathrm{eV}$, too weak to induce a difference in the absorption profile. Both codes give similar results for the constant density case, and our TITAN code can be used safely to explain WA features in this study in spite of its smaller number of lines treated.

The influence of the physical parameters is illustrated in the following figures, which display a few examples of pure absorption spectra. In order to easily see the influence of the absorption on the soft X-ray excess, we have computed all models with simple power law incident continua $\left(F_{v} \propto v^{-\alpha}\right)$.

Figure 3 illustrates the influence of the primary continuum energy range. The thin lines correspond to a primary continuum from 0.1 to $20 \mathrm{keV}$ (actually the Gierliński-Done model), and the thick lines show the corresponding results for a primary continuum covering the $0.01-100 \mathrm{keV}$ range. Both models 

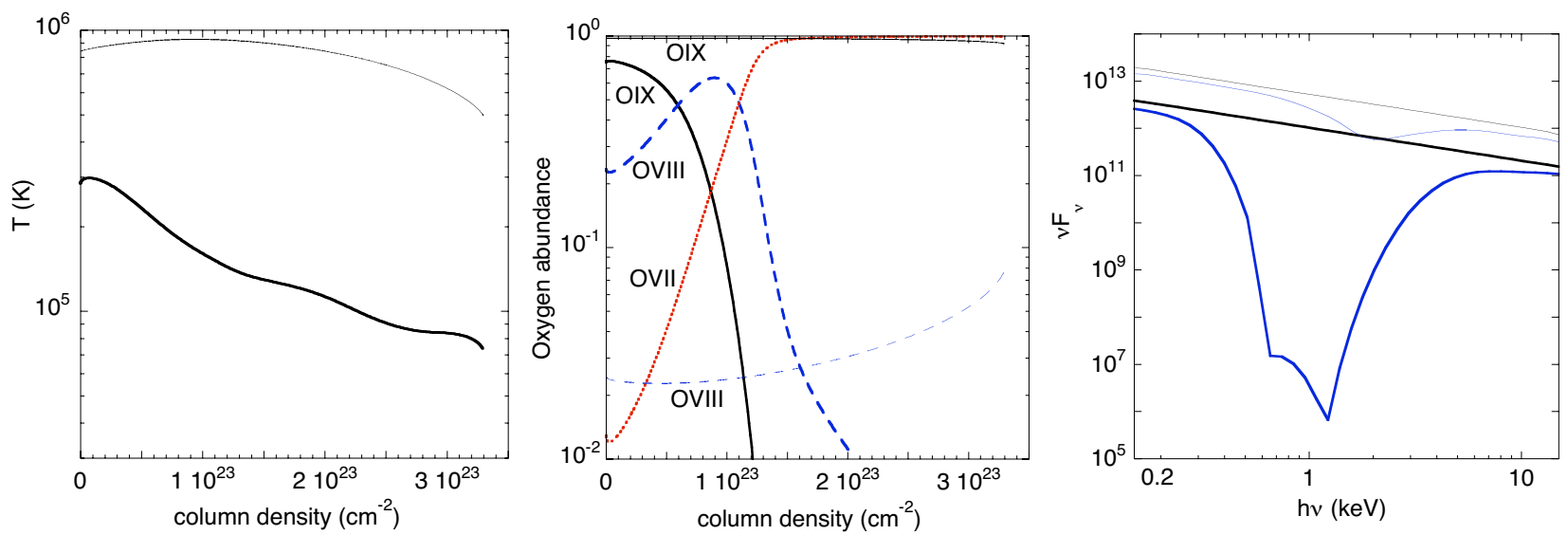

Fig. 3. Influence of the energy range of the primary continuum. The thin lines correspond to a primary continuum in the range $0.1-20 \mathrm{keV}$, and the thick lines show the corresponding results for a primary continuum in the range $0.01-100 \mathrm{keV}$. Both models have the same parameters as Fig. 2 (note that the ionization parameter is integrated over the whole continuum, as explained in the text; this induces a slightly larger flux level for the "truncated" continuum). For both models, we give the temperature profile (left panel), the fractional ionization abundances of oxygen (middle panel, including O VII to O IX for the "extended" continuum and O VIII and O IX - which dominates - for the "truncated" continuum) and the incident and absorption spectra (right panel). The spectral resolution is 2 and fluxes are in arbitrary units.

have the same density, same column density, same metal abundances, same microturbulent velocity, and same ionization parameter (integrated over the whole continuum, which explains why the flux level is slightly higher for the "truncated" continuum). The panel on the left shows the temperature profile: it is much higher for the "truncated" continuum. Indeed with the "extended" continuum the model includes more species able to cool the medium, as it is seen in the middle panel, which displays the fractional ionization abundances of oxygen for both models. For the "truncated" continuum oxygen is mainly in the form of OIX, while O VII and O VIII are predominant for the "extended" continuum. As a result there is a strong imprint of the absorption in the case of the "extended" continuum, as it can be seen in the panel on the right where the spectra are displayed with a spectral resolution of 2 .

Figure 4 shows a few examples of absorption spectra. They are not aimed to illustrate in detail the influence of the different physical parameters, but only to show the variations from one spectrum to the other. All these models were calculated at a constant density. The top panels correspond to a primary continuum with $\alpha=1$, and the panels on the bottom to continua with $\alpha$ larger than unity. The abundances are cosmic except when mentioned otherwise. All primary continua extend from 0.01 to $100 \mathrm{keV}$ except when indicated (in the following we shall call the "standard" primary continuum the $\alpha=1$ power law within these limits). Among the top panels, the left one shows the influence of the ionization parameter, the middle one the influence of the column density, the right one the influence of the abundances. The bottom panels show the influence of the column density, the ionization parameter and the limits of the incident continuum. One can see that all absorption spectra have a trough around $1 \mathrm{keV}$. Its position is shifted towards slightly larger energies for higher values of the ionization parameter, as more ionized species become dominant.

\subsection{A fine tuning prescription for pure absorption models: total pressure equilibrium}

A conclusion which can be drawn from Fig. 4 is that the intensity of the trough varies strongly with the values of the parameters. Indeed several spectra of this figure would be completely incompatible with any observed X-ray spectrum, because the absorption is much too large, although all parameters vary in only relatively small ranges. As a consequence, any small variation with time of the column density on the line of sight, or of the flux of the primary continuum, would induce a strong variation on the shape of the X-ray spectrum. This implies that there must be some kind of "fine tuning" which insures that the intensity of the trough cannot exceed a given value.

The previous models have been computed assuming, as it is generally done in this kind of problem, that the absorbing medium is one or several slabs of constant density. This is not necessarily appropriate, due to the relatively short dynamical time scales of the absorbing gas. We will see in the last section that the pure absorption hypothesis implies a small distance between the primary source (or the black hole) and the absorber. The dynamics of the absorbing medium as a whole would thus be dominated by the gravitation of the black hole, and its dynamical time $t_{\mathrm{dyn}}$ would be of the order of the orbital time at the distance $R$ of the black hole:

$t_{\mathrm{dyn}} \sim \frac{R}{v_{\mathrm{abs}}} \sim \frac{1}{\Omega}=5 \times 10^{4} r_{10}^{3 / 2} M_{7} \mathrm{~s}$,

where $v_{\mathrm{abs}}$ is the typical velocity of the absorber (rotation, outflowing velocity, large scale turbulence, etc.), $\Omega$ is the angular velocity, $r_{10}$ the distance to the black hole expressed in units of $10 R_{\mathrm{G}}$, and $M_{7}$ is the mass of the black hole in units of $10^{7} M_{\odot}$.

Note that the other important time scales (heating and cooling times, ionization and recombination times) are much 

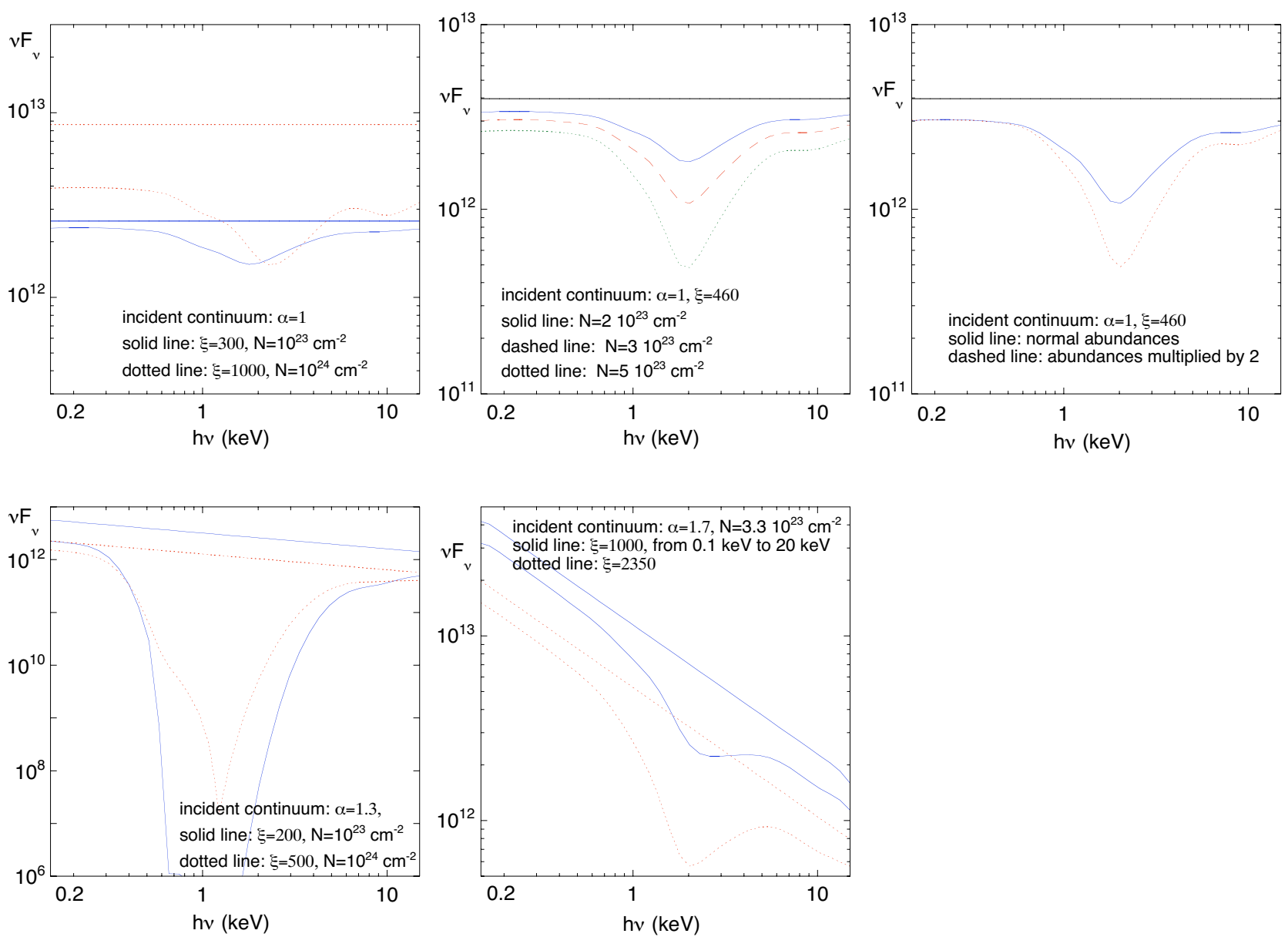

Fig. 4. Incident and absorption spectra for various constant density models, displayed with a spectral resolution of 2 for different models. The top panels correspond to a primary continuum with $\alpha=1$, and the panels on the bottom to continua with $\alpha$ larger than unity. The abundances are cosmic and the primary continua extend from 0.01 to $100 \mathrm{keV}$ except when it is explicitely mentioned. Fluxes are in arbitrary units.

shorter due to the high gas and radiation density in these central regions (for a discussion, see for instance Collin et al. 2003). So if any perturbation of the pressure equilibrium is produced, another equilibrium state will be established in less than a day for $r_{10} \sim M_{7} \sim 1$. It might not correspond to a constant pressure. If the dynamical behaviour of the absorbing medium is dominated by rotation, it could be close to the hydrostatic equilibrium of an accretion disk in the gravitation potential of the black hole. If it is dominated by outflowing motions it would be a dynamical equilibrium.

As a working approximation, let us assume that the medium reaches a static pressure equilibrium state determined by the total pressure, taken as the sum of the radiation pressure and the thermal gas pressure.

An important aspect of pressure - or hydrostatic - equilibrium models is that the thickness of the illuminated slab cannot exceed a maximum value for a given ionization parameter (Różańska et al. 2006). In a constant density slab, the temperature and the ionization states of the various elements decrease slowly with increasing depth, owing to the absorption of the incident continuum and the re-emission of a diffuse soft X-ray spectrum. When the column density is very large (say larger than $10^{24} \mathrm{~cm}^{-2}$ ), the back side of the slab eventually becomes cold and neutral, and the whole X-ray spectrum is absorbed, allowing us to see only optical and IR photons and, at the other extreme of the spectrum, gamma-ray photons. The object would thus be "Compton thick", and would require a different study.

The behaviour of a slab in total pressure equilibrium is completely different, as the illumination by X-rays induces a thermal instability beyond a given layer in the slab. The phenomenon is due to the $\mathrm{S}$-shape of the temperature versus the radiation to gas pressure ratio, allowing the existence of two or even more stable phases for the same gas pressure. At a given gas pressure, which depends on the energy distribution of the specific intensity, the gas can be in three states of thermal equilibrium, corresponding to a hot and a cold stable solution, and to an unstable intermediate solution. Sometimes there are even five states with three stable ones and two intermediate unstable ones. This was shown by Krolik et al. (1981) for an optically thin gas. In our case the phenomenon is slightly different, as the shape of the radiation spectrum and the radiation pressure itself depend on the location in the medium. In the deepest layers, close to the back surface of the slab, the radiation spectrum becomes harder, and contains only hard X-rays, and as a consequence the $\mathrm{S}$-shape curve is more pronounced, inducing an 

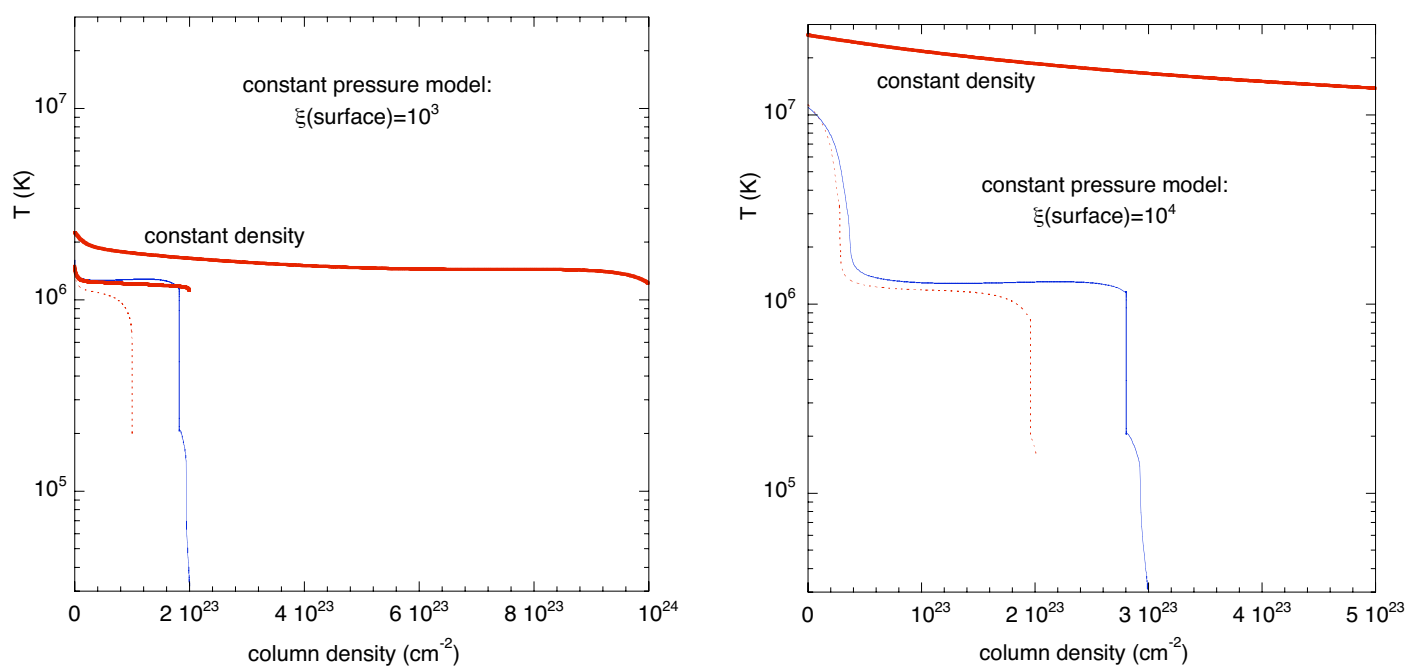

Fig. 5. Temperature profiles for several constant total pressure models (thin lines) computed with the standard primary continuum and two values of the ionization parameter at the surface, $\xi=10^{3}$ and $\xi=10^{4}$. The solid lines correspond to the maximum thickness, while the dotted lines correspond to models with a smaller thickness. The figures also show the temperature profile for constant density models (thick lines) with the same values of the ionization parameter and of the surface density.
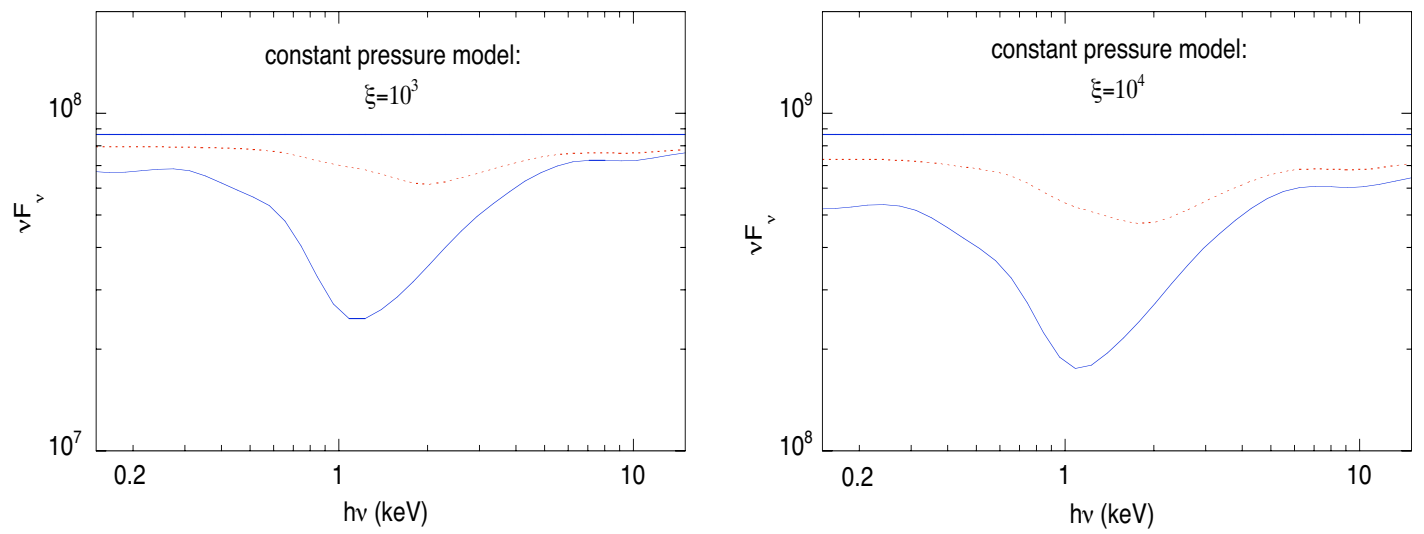

Fig. 6. Incident and absorption spectra corresponding to the constant total pressure models of Fig. 5, displayed with a spectral resolution of 2. The solid lines correspond to the maximum thickness and the dotted lines to a smaller thickness. The straight lines correspond to the primary continuum. Fluxes are in arbitrary units.

important instability and producing a very strong jump in temperature when the gas adjusts to the cold solution.

The thermal instability problem was already discussed in the context of the TITAN code and of hydrostatic equilibrium by Różańska et al. (2002), so we will just recall it here. It is difficult to know in which state (hot or cold) the gas can be when both are allowed. It probably depends on the previous history of the medium. Taking into account conductivity effects can help to solve the problem (Różańska \& Czerny 2000), but it is a very difficult task when conductivity should be coupled with a complete transfer treatment like that of TITAN.

The present scheme of TITAN keeps the density constant in a given layer while searching for the equilibrium temperature. Such a numerical scheme produces a unique but approximate solution (intermediate between the hot and the cold solutions) in the unstable region. We are thus developing a new algorithm allowing one to choose between the hot and the cold exact stable solutions. Even with the present scheme the approximate computation is possible only when the thermal instability is not too pronounced. When the temperature drops suddenly to very low values (of the order of $10^{4} \mathrm{~K}$ ), the radiation pressure becomes dominated by spectral lines and induces a numerical instability which corresponds to a real thermal instability. Thus the slab should necessarily be broken into cold dense clumps, possibly embedded in a warm dilute medium, and it does not exist any longer as an entity. However, as in the constant density case, a cold region completely absorbing the primary source can exist beyond this instability.

A consequence of this maximum thickness of the slab is the existence of a "maximum absorption trough", which cannot be exceeded. Smaller values of the trough can be obtained if the column density is smaller. In this case there is also a decrease of temperature at the back of the slab associated with an absorption, but the drop of temperature is smaller. The decrease 


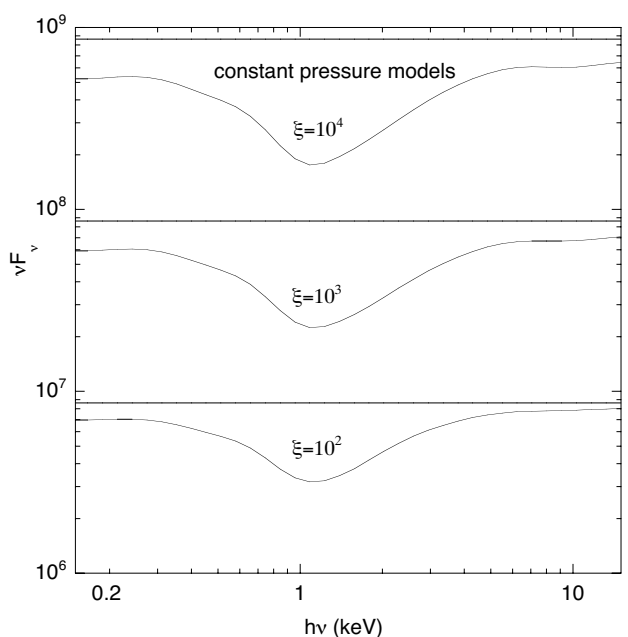

Fig. 7. Absorption spectra for 3 constant total pressure models which have the maximum possible thickness for their given ionization parameter. The straight lines represent the primary continuum. The spectra are displayed with a spectral resolution of 2 . Fluxes are in arbitrary units.

occurs also in constant density models near the back surface, as the radiation can escape more easily and the medium cools. Simply it is more pronounced in constant total pressure models because as the radiation pressure decreases it induces an increase in the gas pressure and therefore in the density, so the medium cools even more rapidly. All these questions will be addressed in more detail in a future paper.

This discussion is illustrated in Fig. 5 which shows the temperature profile for several constant total pressure models (the thin lines). They have been computed with our standard primary continuum. The left panel corresponds to an ionization parameter at the surface $\xi=10^{3}$ and the right one to $\xi=10^{4}$. The solid lines correspond to the maximum thickness, and the dotted lines to models where the thickness has been imposed. In all cases the temperature decreases abruptly close to the back side, and has almost the same profile for the two ionization parameters. For comparison the two panels show also the temperature profiles for constant density models (the thick lines), with the same values of the ionization parameter and of the surface density. One sees that the temperature is almost constant and always high when the density is constant. We have added a constant density model with a very large column density $\left(10^{24} \mathrm{~cm}^{-2}\right)$ to show that the temperature stays high for a larger thickness than the constant total pressure models. Note also that the thicker the medium, the higher the temperature, due to the increased heating by radiation "returning" from the back (cf. Dumont et al. 2000).

Figure 6 displays the incident and absorption spectra for the same constant total pressure models. The solid lines correspond to the maximum thickness and the dotted lines to a smaller thickness. Though the shape of the trough depends on the thickness of the slab, we see that its strength cannot exceed a given value which does not seem to depend on the ionization parameter. Considering only constant pressure models with maximum thickness, one can see that the shapes of the absorption spectra are very similar (cf. Fig. 7). In particular the absorption trough is located at the same energy, around $1 \mathrm{keV}$. Note however that the absorption with respect to the primary continuum is more important at all frequencies for a larger ionization parameter, and consequently a larger column density; this is because the overall spectrum goes down due to the loss of photons through electron scattering. Unfortunately this effect cannot be directly measurable since the primary continuum is not observed.

It is easy to understand the similarity of the absorption spectra. One can see in Fig. 5 the temperature profiles of constant total pressure slabs with the maximum thickness, for $\xi=10^{3}$ and $10^{4}$. The temperature profiles are very similar near the back surface. This is due to the fact that the gas enters a multiple phase regime when the radiation spectrum has a given shape, whatever the physical state of the previous layers. This is well illustrated by Fig. 8 which shows the fractional ionic abundances of oxygen near the back surface, for the constant pressure models corresponding to the maximum thickness, for $\xi=10^{2}, 10^{3}$ and $10^{4}$, as a function of the column density in the slab. The abscissae range corresponds exactly to the same total thickness of $2 \times 10^{22} \mathrm{~cm}^{-2}$. One can see that the fractional abundances vary similarly, although the range of $\xi$ is quite large. This region is dominated by O VII and O VIII ions which contribute to a large fraction of the absorption around $1 \mathrm{keV}$. The same result is obtained for the other elements, which explains why the absorption spectra are almost identical (cf. Fig. 6). Therefore the most important difference between constant density and constant total pressure models lies in the fact that the "intermediate temperature layer" containing highly absorbing species has the same thickness whatever its ionization parameter at the illuminated surface.

With the computed absorption spectra, one can determine some parameters easily deduced from the observed spectra. We have chosen: 1) an "average" photon spectral index $\Gamma_{\mathrm{X}}$ measured from 2 to $10 \mathrm{keV}$ and 2) the ratio of the $0.5 \mathrm{keV}$ flux to the extrapolation of this power law (Ratio).

For different constant pressure models, Fig. 9 shows the variation of $\Gamma_{\mathrm{X}}$ with the column density, for several values of the primary continuum spectral index $\alpha$ and of the ionization parameter at the illuminated surface $\xi$. Since the value of $\Gamma_{\mathrm{X}}$ tends towards that of the primary continuum $\alpha$ (primary continuum) +1 for very thin slabs (because the absorption spectrum is identical to the primary one), we have extrapolated the curves taking this constraint into account. Figure 10 displays several curves giving Ratio versus $\Gamma_{\mathrm{X}}$ : they correspond to different values of $\alpha$ and of $\xi$. The main variable is $\alpha$ and the curves corresponding to different values of $\xi$ are almost aligned. This means that it would not be possible to find a unique fit for an observed spectrum on the basis of these two parameters only.

Nevertheless, helped by the curves of Fig. 9, we have tried to fit some observed spectra with constant total pressure models. Figure 11 shows a comparison on PG 1307+085. The observed spectrum is the result of the "best fit" of the EPIC data of $X M M$ with phenomenological models including blackbodies and power laws, obtained by Piconcelli et al. (2005, see their Fig. 2). We measure $\Gamma_{X}=1.5$ for this object. For clarity we divided the spectra by a power law with this value of $\Gamma_{\mathrm{X}}$. The 

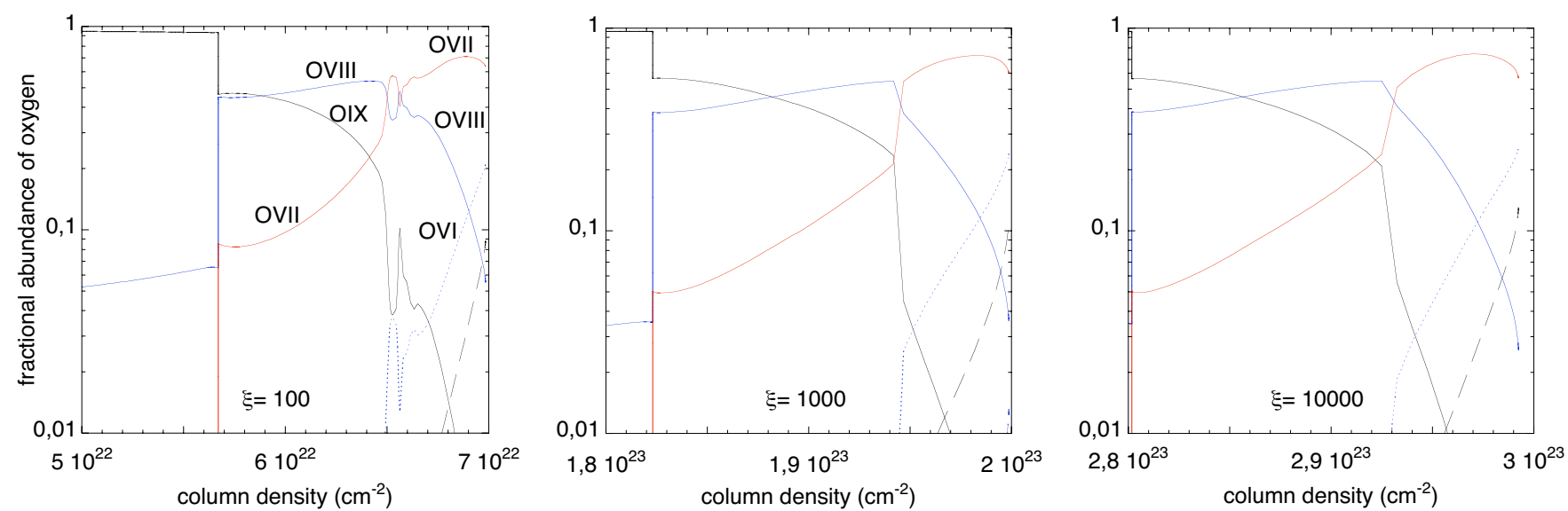

Fig. 8. Fractional ionic abundances of oxygen near the back surface, as a function of the column density in the slab, for the three models of Fig. 7. The abscissae range corresponds to exactly the same total thickness of $10^{22} \mathrm{~cm}^{-2}$. The fractional abundances vary very similarly, although the range of $\xi$ is quite large, and consequently the O VII and O VIII layers are located at very different distances from the illuminated sides.

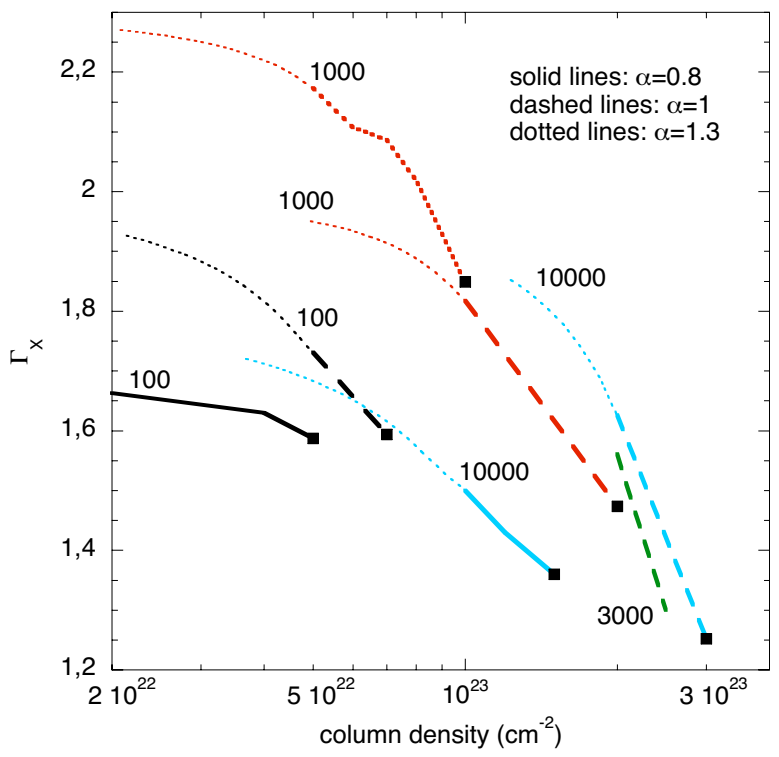

Fig. 9. Value of $\Gamma_{\mathrm{X}}$ measured from 2 to $10 \mathrm{keV}$ on the computed absorption spectra for constant total pressure models, as a function of the column density, for 3 values of the spectral index of the primary continuum ( $\alpha=0.8,1,1.3$ for solid, dashed and dotted lines respectively) and 4 values of the ionization parameter labelled on the curves $\left(\xi=10^{2}, 10^{3}, 3 \times 10^{3}\right.$ and $\left.10^{4}\right)$. The squares mark the position of the maximum column density corresponding to a given ionization parameter. The small dashed lines are extrapolations that take into account that the upper value of $\Gamma$ for optically thin slabs is equal to $\alpha+1$.

model is a constant total pressure slab illuminated by a power law continuum with $\alpha=0.9, \xi=10^{4}$, and $N=2 \times 10^{23} \mathrm{~cm}^{-2}$. The absorption spectrum has been divided by the same power law as the observed spectrum and they are displayed with a spectral resolution of 2 (that corresponds to a dispersion velocity equal to $c / 5$ ) and 100 . This spectrum is well fitted, considering that the narrow emission feature around $0.5 \mathrm{keV}-$ the O VII complex - must be provided by another emitting region.

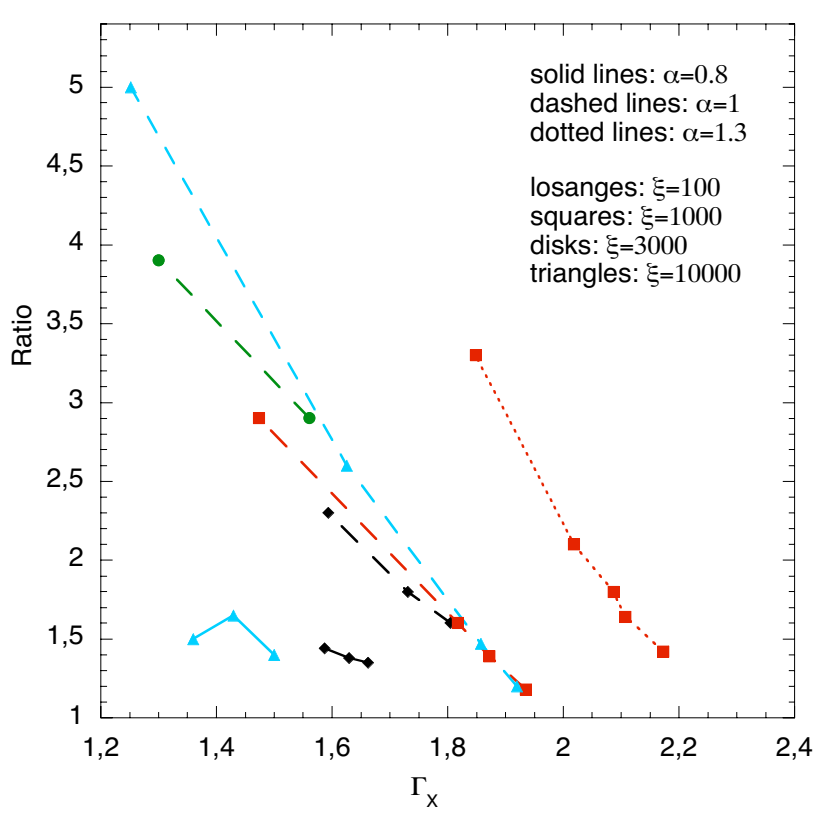

Fig. 10. Ratio of the $0.5 \mathrm{keV}$ flux to the extrapolation of the $\Gamma_{\mathrm{X}}$ power law measured from 2 to $10 \mathrm{keV}$ on the computed absorption spectra versus $\Gamma_{\mathrm{X}}$, for different values of the spectral index of the primary continuum $\alpha$ and different values of $\xi$.

Note that this model corresponds to a thickness smaller than the maximum value allowed for its ionization parameter.

As we can see, it is thus difficult to simultaneously obtain a strong X-ray excess and a relatively flat $2-10 \mathrm{keV}$ slope (i.e. not increasing too steeply). Moreover, a "wiggle" always appears in the 1-12 keV spectrum, due to the decrease of absorbing species above $2 \mathrm{keV}$ and to the presence of iron edges above $7 \mathrm{keV}$. Therefore, a pure power law cannot be obtained in spite of the considerable smearing. With a dispersion velocity smaller than $v / c=0.2$, several other "wiggles" are apparent. To illustrate the effect of the large dispersion velocity, the spectrum is also displayed in Fig. 11 with a spectral resolution of $100\left(F W H M \sim 1300 \mathrm{~km} \mathrm{~s}^{-1}\right)$, corresponding typically to the 

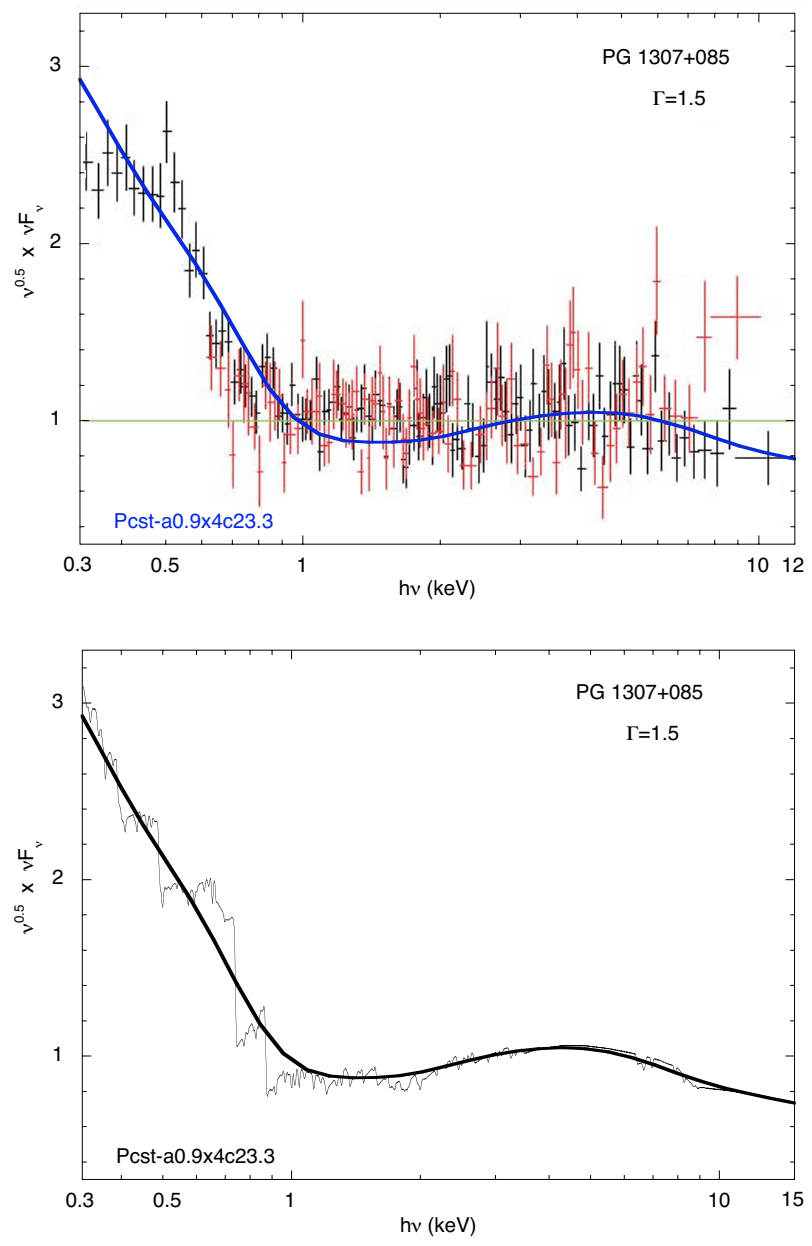

Fig. 11. Comparison between the observed and computed X-ray spectra for PG 1307+085, from the sample of Piconcelli et al. (2005, see their Fig. 2). The model is a constant total pressure slab illuminated by a power law continuum with $\alpha=0.9, \xi=10^{4}$ and $N=2 \times 10^{23} \mathrm{~cm}^{-2}$. Top panel: the computed spectrum is displayed with a spectral resolution of 2. The observed and computed spectra have been both divided by a power law of photon index $\Gamma=1.5$ (arbitrary units). Bottom panel: the computed absorption spectrum of the top panel is displayed with a spectral resolution of 2 (thick solid lines) and 100 (thin solid lines). The spectra have been divided by the same power law. It is extended up to $15 \mathrm{keV}$.

velocities in the Broad Line Region and of a classical WA. It allows one to see the numerous features smeared by the assumed gas motions. Those "wiggles" become apparent with a resolution higher than 5 (i.e. a dispersion velocity less than $0.08 c$ ). It is clear that a smaller velocity dispersion would be unable to account for the smoothness of the observed spectra.

Note that a better fit could be obtained by summing the contribution of two or more absorbing media, since one would then add two degrees of freedom for each new model $(\xi$ and the column density). The impact on the absorption spectrum depends also on the disposition of the absorbers: if they completely cover the primary source and are located at different distances from the source, the external absorber will be illuminated by an already absorbed spectrum; if they are mixed together, they will receive the same incident spectrum.
The degree of freedom would be increased, but the constant total pressure requirement would still limit the strength of the $\mathrm{X}$-ray absorption.

Can we conclude that the pure absorption hypothesis can account for the soft X-ray excess in AGN? We tried to fit other objects from the Piconcelli et al. (2005) sample, without an agreement as good as with PG $1307+085$. There are at least two reasons for this:

- our grid of models is restricted, and does not take into account all possible combinations of parameters;

- two observational parameters are insufficient to determine the shape of the whole X-ray spectrum.

Our purpose in further work is to extend the grid of models and to define several other observational parameters, in order to get (through $\chi^{2}$ tests) a unique and good solution for each object. Presently, although we can already state that - at the present uncertainty level of the observations, and in the range of energy from 0.3 to $10 \mathrm{keV}$ - some spectra can be accounted for by pure absorption constant total pressure models, it is not clear if all spectra could be accommodated for such models.

\section{Emission, partial covering and reflection}

\subsection{Low column density: emission and partial covering}

Until now, we have considered pure absorption spectra, assuming that the emission produced by the medium surrounding the primary source is negligible. This is true only if its covering factor and/or its column density are small. A small covering factor would be in contradiction with the high occurence of soft X-ray excess, assumed here to be caused by the absorbing medium. It would thus be extremely unlikely that a large proportion of quasars and Seyfert nuclei display such an absorption, and that the absorbing medium would be confined to the line of sight of the primary source. Moreover, it is expected that the "emission" coverage factor is about the same as that of the "absorption" one, unless the medium has a very peculiar geometry. Thus, the absorbing medium should surround the source with a coverage factor of the order of unity. In this case, it does not only absorb, but also emits in the UV and soft X-ray range, according to the temperature reached by the deep layers close to the back (non-illuminated) surface.

This emission can be important for relatively thick media illuminated by an intense radiation field. Even for a relatively thin model, the "total" spectrum including the emission (computed assuming that the absorbing medium is spherically distributed around the source with a coverage factor of unity) is slightly more intense than the pure absorption one. They would be more different in the absence of the strong smearing, since several narrow spectral features would be present in emission in the total spectrum, while they are absent in the pure absorption spectrum. With a resolution of 2 , the difference between the two spectra would not be detectable, since the primary continuum itself is not observed. 

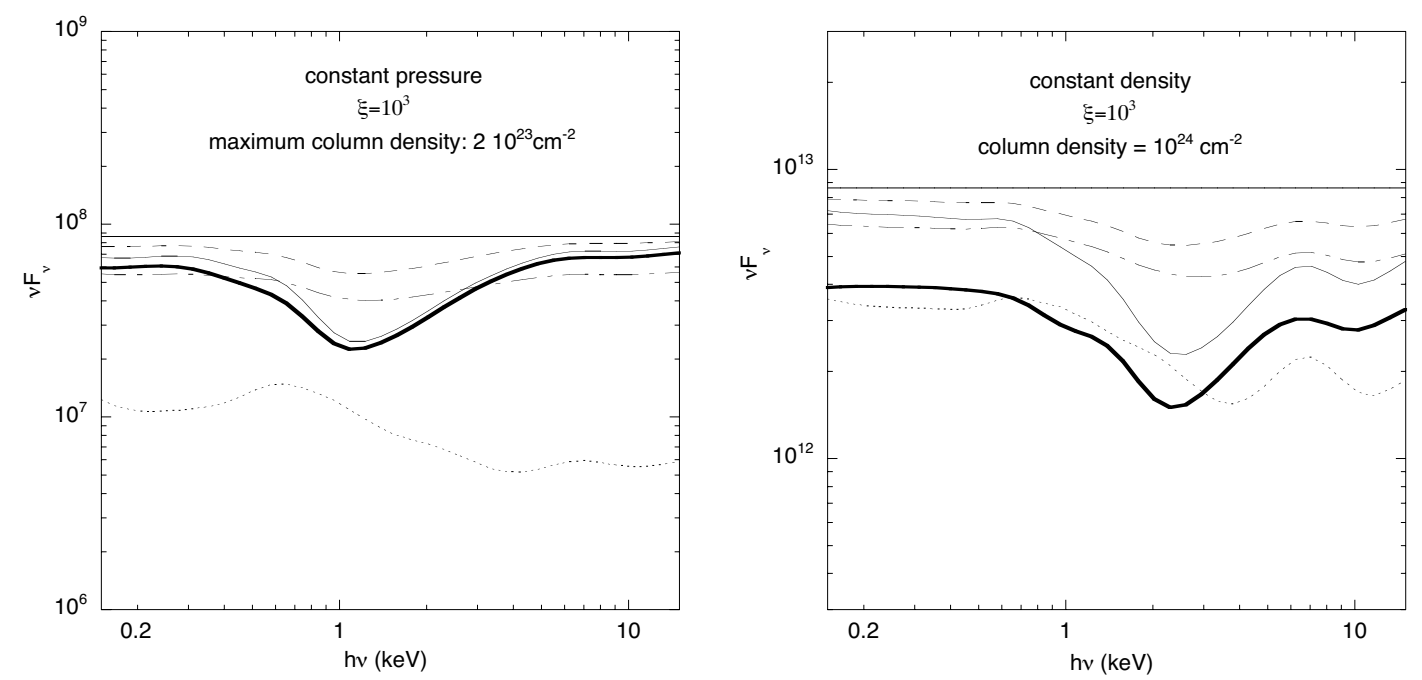

Fig. 12. Different combinations of the absorbed, emitted and reflected spectra are displayed with a spectral resolution of 2 , for a constant total pressure model which has the maximum possible thickness for its given ionization parameter, $2 \times 10^{23} \mathrm{~cm}^{-2}$, and for a constant density model with a larger column density and the same ionization parameter. Thick solid lines: pure absorption spectrum. Thin solid lines: absorption + outward emission spectra, assuming that the absorbing medium is a spherical envelope around the primary source. Dotted lines: reflection spectrum. Dashed lines: combination of equal proportions of primary and absorption spectra (i.e. partial coverage of the line of sight equal to 50\%). Large and small dashed lines: combination of equal proportions of primary, reflection and absorption spectra. The straight lines represent the primary continuum. Fluxes are in arbitrary units.
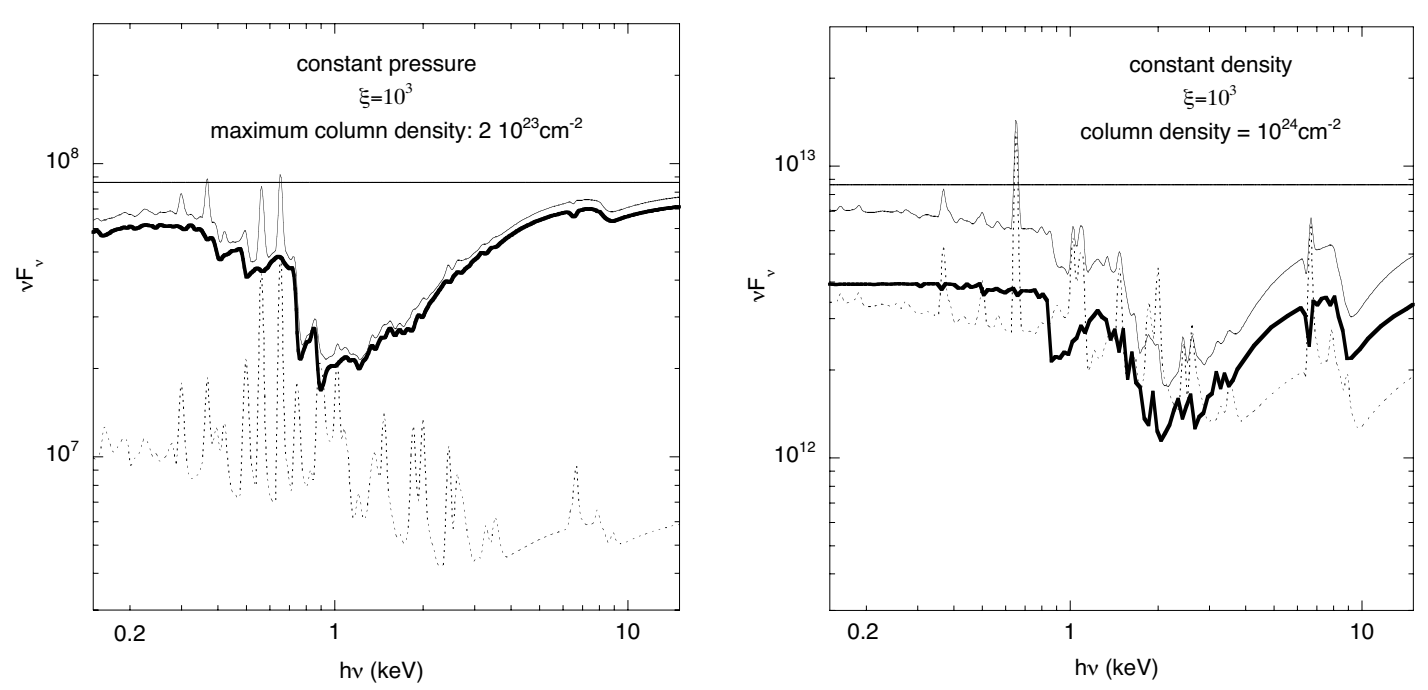

Fig. 13. The spectra corresponding to the same models as in Fig. 12 but displayed with a spectral resolution of 30. Thick solid lines: pure absorption spectrum. Thin solid lines: absorption + emission spectrum, assuming that the absorbing medium is a spherical envelope around the primary source. Dotted lines: reflection spectrum. The straight lines represent the primary continuum. Fluxes are in arbitrary units.

Figure 12 shows two examples of the influence of the emission, assuming that the absorbing medium is spherically distributed around the source with a coverage factor of unity. The left panel shows the spectrum of a constant total pressure model with its maximum thickness. Since this thickness is limited to a low value, the difference between the total and the pure absorption spectrum is small. The right panel shows the spectrum of a constant density medium with a large column density. Here the total spectrum differs appreciably from the pure absorption spectrum, and the emission should be taken into account in the fitting procedure. Figure 13 displays the same spectra but with a velocity dispersion equal to $c / 75$ (corresponding to a spectral resolution of 30). Now the differences between the emission and absorption spectra appear distinctly, as the emission spectrum contains several intense emission lines which are not present in the pure absorption spectrum. 


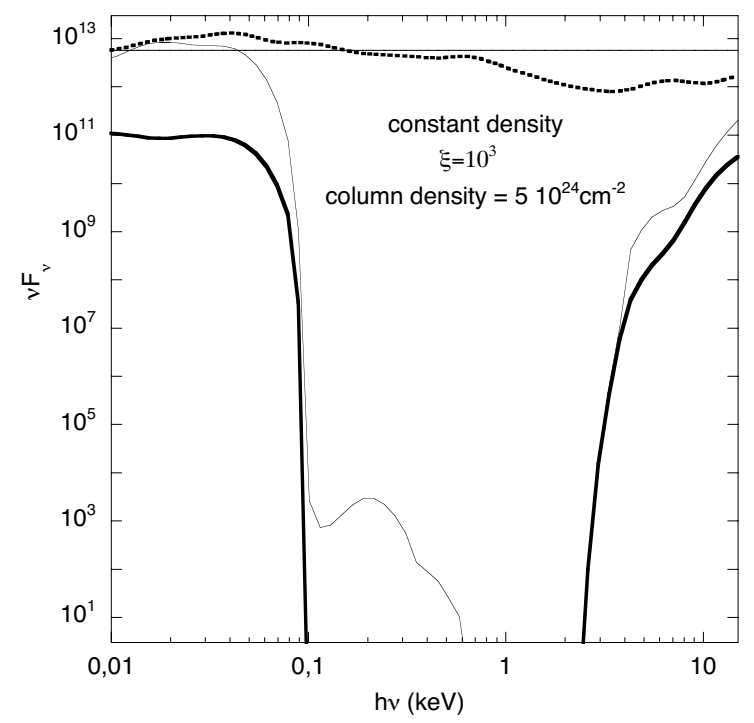

Fig. 14. Spectrum for a constant density thick model, and the standard primary continuum displayed with a spectral resolution of 2 . Thick solid lines: pure absorption spectrum. Thin solid lines: absorption + emission spectrum, assuming that the absorbing medium is a spherical envelope around the primary source. Thick dotted lines: reflection spectrum. The straight lines represent the primary continuum. Fluxes are in arbitrary units.

Another effect not considered so far is a partial and not complete coverage factor of the absorbing medium. This possibility offers an enormous degree of freedom for the shape of the spectrum, and in particular it can erase efficiently the large absorption trough created in the pure absorption models. Figure 12 shows (in dashed lines) the result of a partial coverage of 50\%; the absorption is almost suppressed. It is clear that keeping the coverage factor as a free parameter would allow fits to all kinds of spectra, when added to the other important parameters, i.e. the spectral index of the primary continuum, the ionization parameter and the column density.

If the primary source is not completely obscured by the absorbing medium we have also to take into account another ingredient in the observed spectrum, namely the reflection spectrum. Since the whole covering factor is smaller than unity, one can possibly see also the side of the absorbing clouds facing the primary source. Of course, it implies that the primary source itself is not opaque, or that it is closely mixed with the absorbing/emitting medium.

\subsection{High column density: reflection models}

Up to now we have considered relatively thin media, whose Thomson thickness is smaller than unity. Let us consider now thick media with a Thomson thickness of the order of or larger than unity (i.e., a column density larger than $10^{24} \mathrm{~cm}^{-2}$ ). If a thick medium covers completely the line of sight of the primary source, nothing except the extreme parts of this continuum (the UV and the Gamma bands) would be seen, as shown in Fig. 14. Here we enter the domain of the "reflection" models,

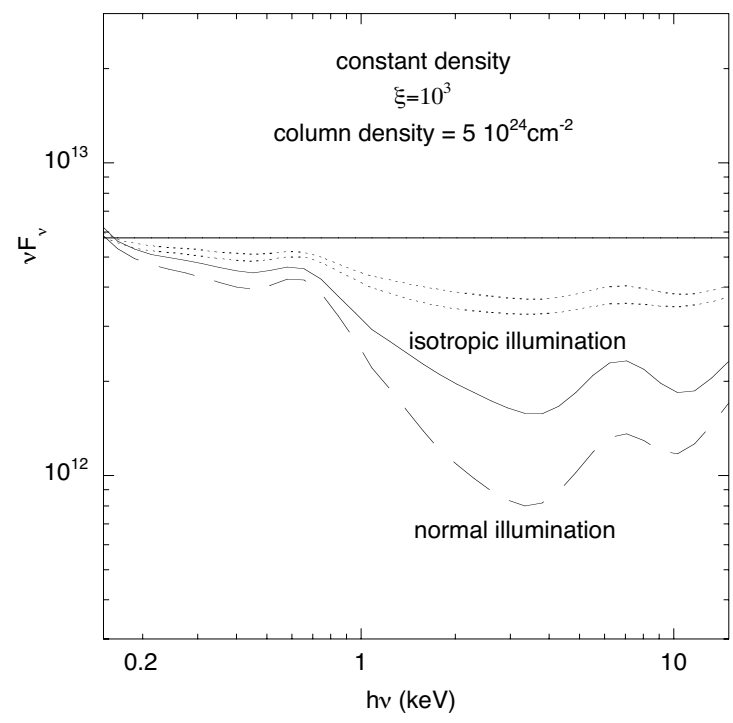

Fig. 15. A zoom on the reflection spectrum for the thick model given in Fig. 14. The solid and long dashed lines show the reflection spectra in the case of an isotropic and a normal illumination, respectively, and the dotted lines shows the "observed spectrum", i.e. the half sum of the reflection and primary spectrum. The straight lines represent the primary continuum. Fluxes are in arbitrary units.

which were invoked to account for the presence of the soft $\mathrm{X}$-ray band.

In almost all the cases considered so far, the reflection spectrum was negligible, as it can be seen in the left panel of Fig. 12, where the reflection spectra are shown, as well as a combination of equal proportions of primary, absorption and reflection spectra. For thick ionized media, reflection completely dominates the transmission and the outward emission. But it is not seen, unless the surrounding medium is located behind or to the side of the primary source. The atmosphere of an accretion disk illuminated from above, either by a central source or by a patchy hot corona (Capriotti et al. 1981; Haardt \& Maraschi 1991, 1993; Ross \& Fabian 1993; Nayakshin et al. 2000; Nayakshin \& Kallman 2001), is considered as the best location for such a reflection. The disk atmosphere is then in hydrostatic equilibrium under the gravity of the central black hole, but it was often represented by a constant density slab (Ross \& Fabian 1993 and subsequent works), or by a slab in total pressure equilibrium (Collin et al. 2003), in both cases heated from below by the viscous release of gravitational energy.

We have run a set of constant density thick models to check whether it is possible to account for the soft X-ray excess in PG quasars by reflection. Instead of the normal illumination considered previously, we assumed an isotropic illumination, which is more realistic if the reflecting medium is located close to the primary source and has a comparable size (it was actually the type of illumination considered in all previous studies performed with the TITAN code). As an illustration, Fig. 15 shows a comparison between the reflection spectra in the case of normal and isotropic illuminations, and we see that the reflection is larger in the isotropic case. This is expected as the mean path of the photons in the normal direction is smaller in 


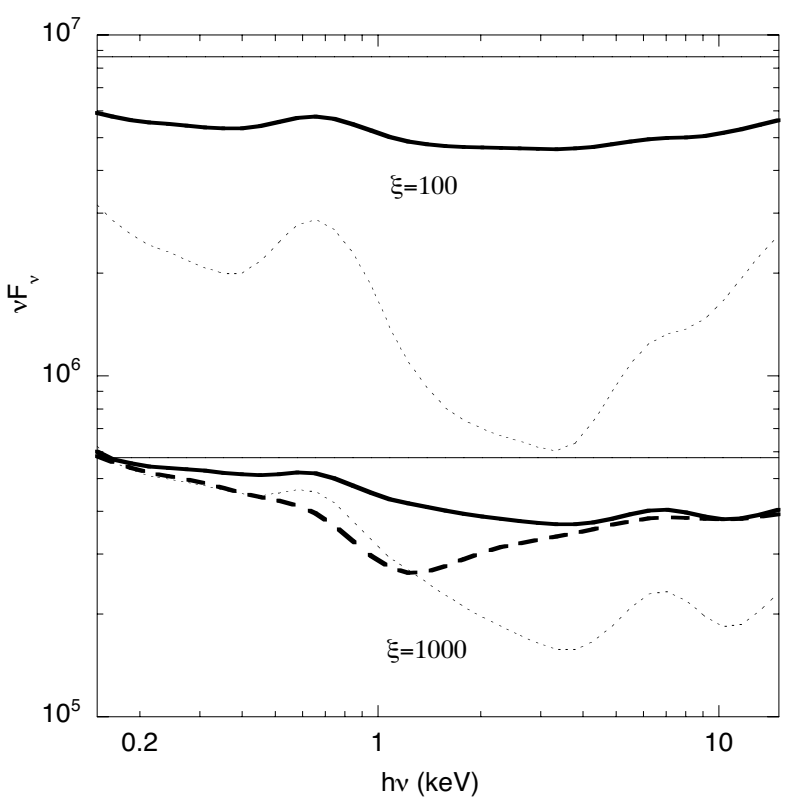

Fig. 16. Computed spectra for thick reflection models for two values of the ionization parameter. The straight lines represent the primary continuum. Thin dotted lines: reflection spectrum. Thick solid lines: half sum of reflection plus primary spectrum. The figure illustrates the fact that the spectrum which reaches the observer (reflection plus primary) never displays a strong X-ray excess. The thick dashed lines show one of the "observed" spectrum, after being absorbed by a constant total pressure slab of column density $10^{22} \mathrm{~cm}^{-2}$ and an ionization parameter $\xi=100$, with a dispersion velocity equal to $0.2 c$. Fluxes are in arbitrary units.

the case of an isotropic illumination than in the case of a normal illumination.

Figure 16 shows two examples of such models, with a small and with a large ionization parameter. It is obvious that when the reflection spectrum is added to the primary continuum, the soft X-ray excess almost disappears, and one understands why it is so difficult, with such models, to account for the large soft excess observed in some PG quasars and in high accretors like NLS1s. We have tried without success to fit some other observed spectra from the Piconcelli et al. (2005) sample, when the primary continuum was added to the reflection one. To summarize the problem:

- either the ionization parameter is small and the reflection spectrum displays a strong X-ray excess, but is negligible compared to the primary one;

- or the ionization parameter is large, and the reflection spectrum is comparable in flux to the primary one, but it has only a very small X-ray excess.

This is why a new disk model has been proposed by Fabian et al. (2002). They consider a disk made of a dilute hot gas creating a hard X-ray spectrum which is not seen directly, and whose radiation is reflected by sheets of dense material formed by disk instabilities. Thus the primary source is hidden and only the reflection spectrum is observed. This is presently the only available reflection model able to explain large X-ray excesses, but unfortunately it is valid only for objects accreting close to the Eddington rate, like NLS1s. An alternative approach to hide the primary continuum could be the effect of "light bending" where a significant fraction of light emitted by the central source is bent onto the disc rather than escaping to the observer (Crummy et al. 2005).

\section{Discussion}

Thus, absorption models seem able to account for some of the soft X-ray excesses, and we do not know whether one could find good fits for the others with a better grid of models. A mixture of absorbing clouds spanning a range of optical thicknesses and ionization parameters, or only partially covering the primary source, would also help to accommodate all observed spectra. On the contrary, reflection models underpredict even relatively modest soft $\mathrm{X}$-ray excesses, unless the primary continuum is hidden.

It is thus important to examine the implications of these models. Both absorption and reflection models require large ionization parameters and large values of the column density. But the most important condition is the smearing of the spectral features by a very large dispersion velocity. We have indeed seen that a velocity dispersion smaller than $c / 5$ cannot account for the smoothness of the observed spectrum. This large velocity implies that:

- either the absorbing/reflecting medium is located close to the black hole and is thus mixed with the primary source; this would be the case of a medium dominated by rotation and turbulent motions, or

- it is located further out, but in this case it is not gravitationally bound to the black hole and it is necessarily in outflowing motion.

A likely solution for the overall X-ray emission of AGN is thus a hot medium emitting a primary hard X-ray continuum, surrounded by or embedded in a system of absorbing/reflecting/emitting photoionized clouds. In this hypothesis, the dominance of the absorption, or the emission, or the reflection spectrum, would be determined by the coverage factor of the primary source by the clouds, and by their thickness. This clumpy medium could be either a wind dominated by outflowing motion, or a thick inhomogeneous accretion flow.

Let us first assume that the high velocity is due to outflowing motion. Note that in this case the constant total pressure models considered so far would no longer be valid, as the medium would be in a dynamical state. So possibly our constraint on the thickness due to the thermal instability would not hold, but it would be replaced by other constraints, such as those due to radiation pressure acceleration, which would also limit the column density of the absorbing matter. Second, the "dispersion velocity" (actually a velocity gradient) would be accompanied by a blueshift of the lines, but it would not appreciably change the shape of the absorption spectrum.

The wind model is actually reminiscent of the recent discovery of several quasars showing extreme absorption properties (in particular blue-shifted highly ionized Fe lines and a 
sharp feature at $7 \mathrm{keV}$ attributed to the $\mathrm{K}$-shell edge), with outflowing velocities of a few tenths of the speed of light, and column densities of the order of $10^{24} \mathrm{~cm}^{-2}$ (Pounds et al. 2003a,b, 2004). The ionization parameter at the surface of this medium is of the order of $10^{3}$, as in our models. The question is raised as to whether these absorbers are a general - but still undetected - feature of AGNs, as the highly ionised gas is hard to observe.

Assuming the stationary outflow to be a relatively thin shell compared to its distance from the central black hole, one can estimate the mass outflowing rate $\dot{M}_{\text {out }}$ :

$\dot{M}_{\text {out }} \sim \Omega f_{\text {vol }} m_{\mathrm{H}} V_{\text {out }} n_{\mathrm{H}} R^{2}$,

where $\Omega$ is the opening angle of the outflowing medium, $f_{\mathrm{vol}}$ is the volume filling factor (if the medium is made of small clumps), $V_{\text {out }}$ the outflowing velocity, $m_{\mathrm{H}}$ the hydrogen mass. Dividing the outflowing rate by the Eddington accretion rate $\dot{M}_{\text {Edd }}$, and using our definition of $\xi=L / n_{\mathrm{H}} R^{2}$, one gets:

$\frac{\dot{M}_{\text {out }}}{\dot{M}_{\text {Edd }}} \sim \frac{\Omega f_{\text {vol }} m_{\mathrm{H}} V_{\text {out }} R_{\text {Edd }} \eta c^{2}}{\xi}$

where $R_{\text {Edd }}$ is the Eddington ratio $L / L_{\mathrm{Edd}}$ and $\eta$ is the massenergy efficiency conversion factor. If we assume that the whole dispersion velocity is due to outflowing motion with an opening angle of the order of $0.5 \times 4 \pi \sim 6.3$ if the number of Seyfert $1=50 \%$ of Seyfert 2, we get:

$\frac{\dot{M}_{\text {out }}}{\dot{M}_{\text {Edd }}} \sim 2 \times 10^{3} f_{\text {vol }}\left[\frac{R_{\text {Edd }}}{0.3}\right]\left[\frac{V_{\text {out }}}{0.2 c}\right]\left[\frac{\eta}{0.1}\right]\left[\frac{10^{3}}{\xi}\right]$.

Equation (4) shows that $f_{\mathrm{vol}}$ should be smaller than $\sim 5 \times 10^{-4}$, in order for the outflow rate not to have an unrealistic value much larger than the Eddington rate.

It is interesting to deduce other physical properties of the absorbing medium. Since $n_{\mathrm{H}} f_{\mathrm{vol}} \Delta R$ is roughly equal to the column density $N$, where $\Delta R$ is the geometrical thickness of this outflow whose base is at distance $R$ from the central regions, and using the constraint $\Delta R / R<1$, we obtain for the distance $R$ :

$R<\frac{f_{\mathrm{vol}} R_{\mathrm{Edd}} L_{\mathrm{Edd}}}{\xi N}$,

or:

$\frac{R}{R_{\mathrm{G}}}<10^{6} f_{\mathrm{vol}}\left[\frac{R_{\mathrm{Edd}}}{0.3}\right]\left[\frac{10^{3}}{\xi}\right]\left[\frac{3 \times 10^{23}}{N}\right]$.

As $f_{\text {vol }}$ should be smaller than $\sim 5 \times 10^{-4}, R / R_{\mathrm{G}}<500$. From our definition of $\xi$, we can also find the value of the density:

$n_{\mathrm{H}}>8 \times 10^{11}\left[\frac{R_{\mathrm{Edd}}}{0.3}\right]\left[\frac{10^{3}}{\xi}\right]\left[\frac{10^{7} M_{\odot}}{M}\right]\left[\frac{500 R_{\mathrm{G}}}{R}\right]^{2} \mathrm{~cm}^{-3}$.

Thus the density should be high, of the order of that of the atmosphere of a standard accretion disk.

On the other hand, the spread of velocity required by the absorption model implies the existence of many clouds on the line of sight, with a smooth velocity gradient up to $0.2 c$ $\left(F W H M=60000 \mathrm{~km} \mathrm{~s}^{-1}\right)$. The thermal velocity of hydrogen nuclei being of the order of $100 \mathrm{~km} \mathrm{~s}^{-1}$ in the gas giving rise to the absorption spectrum, this means that at least
600 clouds on the line of sight are required to give a dispersion velocity equal to $0.2 c$. The ratio $f_{\mathrm{vol}} / f_{\mathrm{cov}}$ is of the order of $r_{\mathrm{c}} N_{\text {los }} / \Delta R=N /\left(n_{\mathrm{H}} \Delta R\right)$, where $r_{\mathrm{c}}$ is the dimension of a cloud, and $N_{\text {los }}$ the mean number of clouds on the line of sight. For $N_{\text {los }}=600$, one gets $r_{\mathrm{c}}<6 \times 10^{8}\left[\frac{N}{3 \times 10^{23}}\right]\left[\frac{8 \times 10^{11}}{n_{\mathrm{H}}}\right] \mathrm{cm}$. Finally, since $f_{\text {cov }} \sim 1$ (primary source completely covered), $f_{\text {vol }} / f_{\text {cov }}$ should be smaller than $5 \times 10^{-4}$ and the following condition should be fulfilled:

$\left[\frac{8 \times 10^{11}}{n_{\mathrm{H}}}\right]\left[\frac{N}{3 \times 10^{23}}\right]\left[\frac{10^{7} M_{\odot}}{M}\right]\left[\frac{500 R_{\mathrm{G}}}{R}\right]>1$.

We see that the inequality (8) is marginally realized. Thus the model is consistent from a phenomenological point of view. However it remains to be justified on physical grounds, especially to seek of a confining mechanism of the small dense clouds (magnetic?). Note that they could be simply transient entities.

As the absorbing medium should be located within $500 R_{\mathrm{G}}$, its dynamics should comprise a fraction of the rotational motion. It could perhaps be identified with the inner part of a radiatively driven wind launched by the disk, as proposed by Murray \& Chiang (1995, and subsequent works). However the wind does not reach relativistic velocities in their model. The high velocities close to the black hole could also be triggered by the accretion disk releasing magnetically driven winds, like those proposed e.g. by Königl \& Kartje (1994). In particular Czerny \& Goosmann (2004) showed that external X-ray heating by magnetic flares can account for vertical acceleration of disk material.

In this computation we have neglected the medium at a larger distance. The stationary assumption indeed implies the presence of matter at any distance from the center. Since the column density of this medium decreases as $R^{-2}$, the material located at, say, $1000 R_{\mathrm{G}}$ would have a column density 100 times smaller than this value inside $100 R_{\mathrm{G}}$ and would therefore be undetectable (or detectable as a "classical" Warm Absorber). Note also that we have assumed a stationary outflow, but of course it would have been equivalent to assume sporadic events, separated by the time (of the order of $R / V_{\text {out }}$ ) that it would take for the absorbing medium to be replenished before its disappearence by dilution.

An alternative solution to the outflow is a thick inhomogeneous accretion flow, whose dynamics is dominated by rotation and turbulent motions. But now the absorbing medium should be located at a distance of the order of $25 R_{\mathrm{G}}$, as it should be gravitationally bound to the black hole and simultaneously have relativistic velocities. This is actually close to the model proposed by Collin et al. (1996), consisting of a quasi-spherical (or a thick disk) distribution of clouds covering almost totally a primary source of X-rays. The difference to the present model lies in the very large column density of the Collin et al. accretion flow (Thomson opacity of the order of 10-100), which did not allow the leakage of any transmitted radiation, so partial covering was required and had to be fine tuned. The interest of this model was to account not only for the X-ray emission, but also for the UV spectrum, due to the outward emission of the optically thick cloud system. In the present model, another UV source is required, like a geometrically thin accretion disk. 
None of the models are very satisfactory from a physical point of view. Due to its large column density, the wind implies too massive outflows. Both the wind and the accretion models require additional UV emission, which has to be provided by a geometrically thin accretion disk. The coexistence of a spherical accretion flow and a thin disk seems quite artificial.

So we would prefer a "hybrid model", including an accretion disk with a hot patchy corona emitting the whole X-ray spectrum, through the "classical" Compton reflection on the disk and inverse Compton process in the corona initially proposed by Haardt \& Maraschi (1991 and 1993), the UV emission being produced by the viscous release in the disk. The emerging spectrum in the $0.1-10 \mathrm{keV}$ range would be similar to the "observed" spectra shown in Fig. 16 and would display a small soft X-ray excess. If such a spectrum is absorbed by a moderately thick wind, the excess would be increased and would become comparable to the observations. As an illustration, Fig. 16 shows one of the "observed" spectra, after being absorbed by a constant total pressure slab of column density $10^{22} \mathrm{~cm}^{-2}$ with an ionization parameter $\xi=100$. Assuming that this slab also has a dispersion velocity equal to $0.2 c$, one gets a spectrum comparable to the observations. The interest of this hybrid model is to require less extreme conditions for the outflowing mass, as the column density and the ionization parameter of the wind are smaller. According to Eqs. (4) and (6), $f_{\text {vol }}$, and thus $\dot{M}_{\text {out }} / \dot{M}_{\text {Edd }}$, would be 300 and 30 times smaller than in the previous absorption model, respectively.

\section{Conclusion}

This study has shown that absorption models could account for some strong soft X-ray excesses, while reflection models are possibly able to account for weak soft X-ray excesses, but certainly not for the large ones (including those of typical PG quasars), unless the primary continuum is hidden from view. An important conclusion is also that pure absorption models require "fine tuning" of the absorber, in order to constrain the $1 \mathrm{keV}$ trough, which otherwise could have any strength. We have suggested a medium in total pressure equilibrium, which leads to a maximum intensity of the trough, as well as a "universal" shape of this maximum trough, due to the thermal instability mechanism. A complete grid of constant total pressure models, very demanding in computation time, is necessary to pursue this study.

In the absorption model, either a thick accretion flow or a relativistic wind is required. Neither of them seems very realistic from a physical point of view, and moreover both models require an additional source of UV emission, such as a geometrically thin accretion disk. Therefore we favor a "hybrid" model, where the primary UV-X source could be produced by a disk-corona system, and then absorbed by a modest relativistic wind.

A clue to the problem could be obtained with observational data from Astro-E2, as this instrument should give good spectra above $10 \mathrm{keV}$. Looking at Fig. 11, where plotting the energy up to $15 \mathrm{keV}$ reveals a well-defined slope, this might bring important limitations. Variability is also a key point. It can help to disentangle the absorption and reflection models, as one would expect different behaviours of the light curves in the hard and soft X-ray bands: in the first case, the hard and the soft X-ray flux should vary simultaneously, while in the reflection model, there should be a time delay between the two light curves.

Acknowledgements. A. C. Gonçalves acknowledges support from the Fundação para a Ciência e a Tecnologia, Portugal, under grant no. BPD/11641/2002. Part of this work was supported by grants 2P03D00322 and 1P03D00829 of the Polish State Committee for Scientific Research, the Laboratoire Européen Associé Astrophysique Pologne-France, and by the Hans-Böckler-Stiftung.

\section{References}

Barvainis, R. 1993, ApJ, 412, 513

Behar, E., Rasmussen, A. P., Blustin, A. J., et al. 2003, ApJ, 598, 232

Blustin, A. J., Page, M. J., Fuerst, S. V., Branduardi-Raymont, G., \& Ashton, C. E. 2005, A\&A, 431, 111

Branduardi-Raymont, G., Sako, M., Kahn, S. M., et al. 2001, A\&A, 365, L140

Capriotti, E., Foltz, C., \& Byard, P. 1981, ApJ, 245, 396

Collin-Souffrin, S., Czerny, B., Dumont, A.-M., \& Zycki, P. T. 1996, A\&A, 314, 393

Collin, S., Coupé, S., Dumont, A.-M., Petrucci, P.-O., \& Różańska, A. 2003, A\&A, 400, 437

Collin, S., Dumont, A.-M., \& Godet, O. 2004, A\&A, 419, 877

Crummy, J., Fabian, A. C., Brandt, W. N., \& Boller, Th. 2005, MNRAS, 361, 1197

Czerny B., \& Zycki, P. T. 1994, ApJ, 431, L5

Czerny, B., \& Goosmann, R. 2004, A\&A, 428, 353

Dumont, A.-M., Abrassart, A., \& Collin, S. 2000, A\&A, 357, 823

Dumont, A.-M., Collin, S., Paletou, F., et al. 2003, A\&A, 407, 13

Fabian, A. C., Ballantyne, D. R., Merloni, A., et al. 2002, MNRAS, 331, L35

Ferland, G. J., Korista, T., Verner, D. A., et al. 1998, PASP, 110, 761

Gallagher, S. C., Brandt, W. N., Chartas, G., \& Garmire, G. P. 2002, ApJ, 567, 37

Gallo, L. C., Tanaka, Y., Boller, Th., et al. 2004, MNRAS, 353, 1064

Gierliński, M., \& Done, C. 2004, MNRAS, 349, L7

Grupe, D., Mathur, S., \& Elvis, M. 2003, AJ, 126, 1159

Halpern, J. P. 1984, ApJ, 281, 90

Haardt, F., \& Maraschi, L. 1991, ApJ, 380, L51

Haardt, F., \& Maraschi, L. 1993, ApJ, 413, 507

Ives, J. C., Sanford, P. W., \& Penston, M. V. 1976, ApJ, 207, L159

Kaastra, J. S., Steenbrugge, K. C., Raassen, A. J. J., et al. 2002, A\&A, 386,427

Kallman, T., \& Bautista, M. 2001, ApJS, 133, 221

Kinkhabwala, A., Sako, M., Behar, E., et al. 2002, ApJ, 575, 732

Königl, A., \& Kartje, J. F. 1994, ApJ, 434, 446

Krolik, J. H., McKee, C. F., \& Tarter, C. B. 1981, ApJ, 249, 422

Laor, A., Fiore, F., Elvis, M., Wilkes, B. J., \& McDowell, J. C. 1997, ApJ, 477, 93

Leighly, K. M., Mushotzky, R. F., Yaqoob, T., Kunieda, H., \& Edelson, R. 1996, ApJ, 469, 147

Magdziarz, P., Blaes, O. M., Zdziarski, A. A., Johnson, W. N., \& Smith, D. A. 1998, MNRAS, 301, 179

Murray, N., \& Chiang, J. 1995, ApJ, 454, L105

Mushotzky, R. F., Serlemitsos, P. J., Boldt, E. A., Holt, S. S., \& Becker, R. H. 1978, ApJ, 220, 790

Netzer, H. 1993, ApJ, 411, 594

Netzer, H. 1996, ApJ, 473, 781

Nayakshin, S., Kazanas, D., \& Kallman, T. R. 2000, ApJ, 537, 833

Nayakshin, S., \& Kallman, T. R. 2001, ApJ, 546, 406 
Nicastro, F., et al. 2000, ApJ, 536, 718

Péquignot, D., et al. 2001, Spectroscopic Challenges of Photoionized Plasmas, ASP Conf. Ser., 247, 247, 533

Piconcelli, E., Jimenez-Bailón, E., Guainazzi, M., et al. 2004, MNRAS, 351, 161

Piconcelli, E., Jimenez-Bailón, E., Guainazzi, M., et al. 2005, A\&A, 432, 15

Porquet, D., Reeves, J. N., O’Brien, P., \& Brinkmann, W. 2004, A\&A, 422, 85

Pounds, K. A., Nandra, K., Stewart, G. C., George, I. M., \& Fabian, A. C. 1990, Nature, 344, 132

Pounds, K. A., Reeves, J. N., King, A. R., et al. 2003a, MNRAS, 345, 705

Pounds, K. A., King, A. R., Page, K. L., \& O’Brien, P. T. 2003b, MNRAS, 346, 1025

Pounds, K. A., Reeves, J. N., Page, K. L., \& O’Brien, P. T. 2004, ApJ, 616,696

Ross, R. R., \& Fabian, A. C. 1993, MNRAS, 261, 74
Różańska, A., \& Czerny, B. 2000, MNRAS, 316, 473

Różańska, A., Dumont, A.-M., Czerny, B., \& Collin, S. 2002, MNRAS, 332, 799

Różańska, A., Czerny, B., Siemiginowska, A., Dumont, A.-M., \& Kawaguchi, T. 2004, ApJ, 600, 96

Różańska, A., Goosmann, R. W., Dumont, A.-M., \& Czerny, B. 2006, A\&A, accepted [arXiv: astro-ph/0512310]

Sako, M., et al. 2003, ApJ, 596, 114

Sambruna, R. M., Netzer, H., Kaspi, S., et al. 2001, ApJ, 546, L13

Sobolewska, M., \& Done, C. 2005, Proceedings for XDAP 2004, AIP to be published

Steenbrugge, K. C., Kaastra, J. S., Sako, M., et al. 2005, A\&A, 432, 453

Tanaka, Y., et al. 1995, Nature, 375, 659

Turner, A. K., Fabian, A. C., Vaughan, S., \& Lee, J. C. 2003, MNRAS, 346,833

Wilkes, B. J., \& Elvis, M. 1987, ApJ, 323, 243

Young, A. J., Lee, J. C., Fabian, A. C., et al. 2005, ApJ, 631, 733 